\title{
The Good and the Bad of Antibiotics
}

\author{
Navdeep S. Chandel ${ }^{\star}$ and G. R. Scott Budinger \\ Department of Medicine, Division of Pulmonary and Critical Care Medicine, Northwestern \\ University Feinberg School of Medicine, Chicago, IL 60611, USA
}

\begin{abstract}
Bactericidal antibiotics with diverse mechanisms of action induce generation of mitochondrial reactive oxygen species in mammalian cells (Kalghatgi et al., this issue).
\end{abstract}

\begin{abstract}
Along with vaccines, antibacterial antibiotics are perhaps the most successful pharmacologic therapy of the modern era. The use of antibiotics has markedly diminished mortality from infectious diseases, reduced the burden of tuberculosis in the modern world, improved survival after trauma, and allowed the development of therapies that intentionally or incidentally suppress the immune system for the treatment of autoimmune diseases and cancer. It is estimated that children in the United States receive on average one prescription for an antibiotic every year and that an antibiotic is prescribed in 15 to $20 \%$ of outpatient visits in adults in the United States (1).
\end{abstract}

Nevertheless, antibiotics have been associated with measureable side effects that tend to be specific for classes of antibiotics sharing a similar mechanism of action. For example, the aminoglycoside antibiotics, which block the bacterial $30 S$ ribosome, are associated with an increased risk of acute kidney injury and ototoxicity, whereas quinolone antibiotics, which block the bacterial DNA topoisomerase II, have been associated with an increased risk of tendinitis. Penicillins, which disrupt the formation of the bacterial cell wall, most commonly induce a potentially severe allergic response. In this issue of Science Translational Medicine, Kalghatgi et al. similarly suggest that increased systemic oxidative stress may represent a hidden cost associated with antibiotic therapy (2).

\section{COLLATERAL DAMAGE}

Antibiotics are intended to kill bacteria. Yet, Kalghatgi et al. found that bactericidal - but not bacteriostatic — antibiotics may also damage healthy mammalian tissues (Fig. 1) (2). The authors found that bactericidal antibiotics with disparate mechanisms of action increased the generation of reactive oxygen species (ROS) in primary mammalian cells in vitro in a concentration- and time-dependent manner. A similar effect was not observed with antibiotics traditionally thought to be bacteriostatic. These results were confirmed in vivo in mice when antibiotics administered in their drinking water resulted in increased markers of oxidant stress in the blood and the mammary glands. Consistent with these findings, the administration of the antioxidant $N$-acetyl-l-cysteine (NAC), which enhances cellular

\footnotetext{
*Corresponding author. nav@northwestern.edu.
} 
antioxidant defenses by increasing concentrations of reduced glutathione, prevented the generation of ROS in response to antibiotics in vitro and oxidative damage to tissues in vivo (Fig. 1). The authors showed that the NAC did not affect the antibacterial activity of the antibiotics in vitro or in mice with Escherichia coli-induced urinary tract infections. A major strength of this study is the confirmation of ROS generation in response to antibiotics with a library of fluorescent dyes, detailed measurements of mitochondrial function, and markers of oxidation of cellular lipids, proteins, and DNA both in cells and in mice (2).

Kalghatgi et al. provide strong evidence suggesting that the mitochondrial electron transport chain (ETC) is the source of ROS in antibiotic-treated mammalian cells. They found that antibiotics that increased ROS generation in cells also inhibited the rate of oxygen consumption in the cells. The authors went on to show that the antibiotics could inhibit the function of key components of complexes I and III of the mitochondrial ETC isolated from bovine mitochondria. These complexes represent the major sites of ROS generation in the mitochondria. In cells lacking functional ETCs [generated by growing them in conditions that result in the progressive loss of mitochondrial DNA ( $\rho_{0}$ cells)], the authors found that the generation of ROS in response to antibiotics was lost.

\section{A MITOCHONDRIAL MECHANISM}

To explain their findings, the investigators invoked the endosymbiotic hypothesis of mitochondrial evolution (3). Mitochondria are thought to have evolved at a single point in evolution when two prokaryotes, an archaeon and a-proteobacterium, developed a symbiotic relationship in which both were mutually dependent on one another for nutritional requirements. Eventually, the achaeon acquired the a-proteobacterium, which became the primordial mitochondria. Since that time, the mitochondria have been subject to continued reductive evolution, in which responsibility for the coding and synthesis of mitochondrial proteins has been increasingly reassigned from the mitochondria to their host nucleus. In humans, plasmid mitochondrial DNA encodes 13 proteins required for electron transport, whereas hundreds of other mitochondrial proteins are encoded by the nuclear genome. According to the endosymbiotic hypothesis, antibiotics targeting fundamental bacterial processes might inadvertently target conserved processes in mammalian mitochondria.

Although the mechanisms by which bactericidal antibiotics interfere with core bacterial functions are well described, it is not clear how interrupting these processes leads to cell death in some bacteria. Kohanski et al. have suggested that mitochondrial ROS generation is a common pathway of cell death induced by a divergent class of bactericidal antibiotics (4). However, two independent groups of investigators have recently questioned this hypothesis in simultaneously published articles in Science $(5,6)$. The authors of those papers found that in vitro bacterial killing by antibiotics is similar under aerobic and anaerobic conditions and failed to find consistent evidence for bacterial ROS generation in response to antibiotics. Kalghatgi et al. confirmed this finding in mammalian cells by showing that the administration of NAC had no effect on bacterial killing in vitro and in vivo (2).

These findings raise important questions as to how bactericidal antibiotics with very disparate mechanisms of action induce mitochondrial ROS in mammalian cells and why 
some antibiotics induce mitochondrial ROS whereas others do not. Indeed, Kalghatgi et al. found that kanamycin and spectinomycin - two aminoglycoside antibiotics with similar mechanisms of action and similar side effect profiles_- had markedly different effects on ROS generation in isolated mammalian cells. The ancestral mitochondrial genome most closely resembles the rickettsial group of a-proteobacteria. Modern rickettsia are sensitive to tetracycline (bactericidal) antibiotics, yet Kalghatgi et al. found that tetracycline did not significantly increase ROS generation in mammalian cells (2). The authors' suggestion that the differences are attributable to whether the antibiotics are bactericidal or bacteriostatic is likely incomplete because this classification is dependent on the organism being examined, the time allowed for killing, and the concentration of the antibiotic used.

\section{ANTIBIOTICS: MORE GOOD THAN HARM}

How should the findings of Kalghatgi and colleagues influence the use of antibiotics? The good news is that the clinically observable toxicity associated with our current widespread use of antibiotics appears to be relatively low when compared with other commonly used drugs. Even in this paper, although the authors showed antibiotic-induced increases in markers of oxidative damage in the blood and mammary glands of treated mice, they did not find evidence of tissue damage or dysfunction. Mammalian cells have abundant antioxidant proteins to scavenge the two major forms of ROS, superoxide anion and hydrogen peroxide, and can rapidly up-regulate these antioxidant proteins in response to oxidative stress, mitigating oxidative damage. In fact, there is very little evidence that oxidative damage plays a major role in prevalent human disease, as illustrated by the consistent failure of antioxidants in clinical trials. It seems likely that these robust antioxidant defenses prevent tissue injury in response to bactericidal antibiotics in most patients. However, in patients with high levels of preexisting oxidative stress, impaired antioxidant defenses, or patients receiving very long courses of antibiotics, antibiotic-induced oxidative stress may become clinically important. Kalghatgi et al. suggest that the coadministration of NAC to these patients might mitigate the injury without adversely affecting bacterial killing.

Although high levels of mitochondrial ROS are damaging, lower levels of ROS might promote beneficial effects such as cellular growth and proliferation (Fig. 1). The mitochondrial ETC allows electrons to leak to molecular oxygen to generate superoxide, which is rapidly converted to hydrogen peroxide to activate cytosolic signaling pathways. We have proposed that these low levels of mitochondrial-generated hydrogen peroxide have persisted through evolution because they serve as signals to indicate the fitness of mitochondrial function before the cell grows, proliferates, or engages in other cellular processes requiring high levels of energy and biosynthetic intermediates (7). Emerging data from our group and others indicate that low levels of mitochondrial ROS are essential for cellular proliferation, differentiation, and metabolic adaptation. Furthermore, low levels of mitochondrial ROS can lengthen the life span of Caenorhabditis elegans and human cells in culture (8). This might explain the observation that prophylactic low-dose antibiotics administered to livestock populations result in an increase in the rates of growth and weight gain, prompting their widespread use as agents to promote growth in commercial animal herds. Although some of this effect is likely due to changes in the gut microbiome (9), it is tempting to speculate that these effects - and perhaps other reported unexpected beneficial 
effects of antibiotics-might result from a low-level increase in the release of mitochondrial ROS.

\section{IMPACT ACROSS THE BOARD}

In conclusion, the study by Kalghatgi et al. is certain to generate controversy with respect to ongoing concerns about antibiotic overuse both in clinical medicine and in agriculture. This controversy will hopefully fuel additional research into how some antibiotics interfere with mammalian mitochondrial metabolism to affect key metabolic signaling pathways or induce oxidative cellular damage. As researchers identify new compounds that exhibit bactericidal properties, they might screen them by examining their effects on mammalian cells grown in media containing galactose instead of glucose. This forces cells to rely exclusively on mitochondrial ATP generation for growth and survival and will quickly identify any compounds that affect mitochondrial function.

Identifying and treating potential off-target effects of antibiotics is important as we face the challenge of increasing antibiotic resistance, particularly the threat posed by drug-resistant strains of common bacteria and extensively drug-resistant Mycobacterium tuberculosis. Successful treatment of these infections is likely to require multiple antibiotics administered over long periods of time, and some of these therapies have already been associated with treatment-limiting side effects (10). Understanding the molecular mechanisms of toxicity may aid in the design of adjunctive therapies to improve antibiotic tolerance.

\section{REFERENCES}

1. Hicks LA, Taylor TH Jr. Hunkler RJ. U.S. outpatient antibiotic prescribing. N. Engl. J. Med. 2013; 368:1461-1462. 2010. [PubMed: 23574140]

2. Kalghatgi S, Spina CS, Costello JC, Liesa M, Morones-Ramirez JR, Slomovic S, Molina A, Shirihai OS, Collins JJ. Bactericidal antibiotics induce mitochondrial dysfunction and oxidative damage in mammalian cells. Sci. Transl. Med. 2013; 5:192ra85.

3. Lane N, Martin W. The energetics of genome complexity. Nature. 2010; 467:929-934. [PubMed: 20962839]

4. Kohanski MA, Dwyer DJ, Hayete B, Lawrence CA, Collins JJ. A common mechanism of cellular death induced by bactericidal antibiotics. Cell. 2007; 130:797-810. [PubMed: 17803904]

5. Liu Y, Imlay JA. Cell death from antibiotics without the involvement of reactive oxygen species. Science. 2013; 339:1210-1213. [PubMed: 23471409]

6. Keren I, Wu Y, Inocencio J, Mulcahy LR, Lewis K. Killing by bactericidal antibiotics does not depend on reactive oxygen species. Science. 2013; 339:1213-1216. [PubMed: 23471410]

7. Sena LA, Chandel NS. Physiological roles of mitochondrial reactive oxygen species. Mol. Cell. 2012; 48:158-167. [PubMed: 23102266]

8. Hekimi S, Lapointe J, Wen Y. Taking a "good" look at free radicals in the aging process. Trends Cell Biol. 2011; 21:569-576. [PubMed: 21824781]

9. Cho I, Yamanishi S, Cox L, Methé BA, Zavadil J, Li K, Gao Z, Mahana D, Raju K, Teitler I, Li H, Alekseyenko AV, Blaser MJ. Antibiotics in early life alter the murine colonic microbiome and adiposity. Nature. 2012; 488:621-626. [PubMed: 22914093]

10. Lee M, Lee J, Carroll MW, Choi H, Min S, Song T, Via LE, Goldfeder LC, Kang E, Jin B, Park H, Kwak H, Kim H, Jeon H-S, Jeong I, Joh JS, Chen RY, Olivier KN, Shaw PA, Follmann D, Song SD, Lee J-K, Lee D, Kim CT, Dartois V, Park S-K, Cho S-N, Barry CE 3rd. Linezolid for treatment of chronic extensively drug-resistant tuberculosis. N. Engl. J. Med. 2012; 367:15081518. [PubMed: 23075177] 


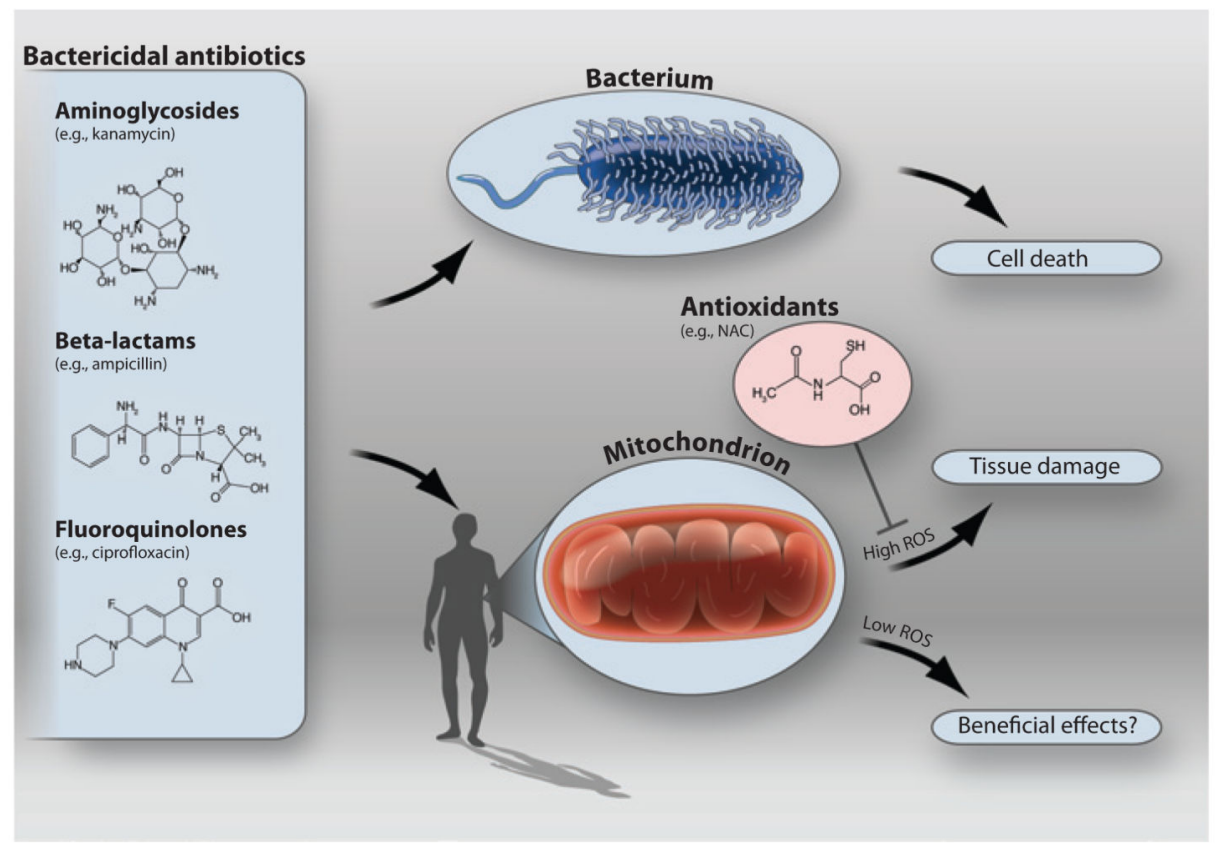

Fig. 1. The good and the bad of antibiotics

Bacteriostatic antibiotics with diverse mechanisms of action are known to induce cell death in bacteria. Kalghatgi et al. report that these antibiotics induced the generation of ROS from mitochondria in mammalian cells and in vivo in mice, which led to tissue damage (2). We also speculate that lower levels of antibiotic-induced mitochondrial ROS might play a signaling role, explaining some of the unexpected beneficial effects of these agents, including organismal growth. 